\title{
ERRATUM
}

Cecilia A. Pérez • Martín R. Carmona •

Juan C. Aravena · Juan J. Armesto

\section{Successional changes in soil nitrogen availability, non-symbiotic nitrogen fixation and carbon/nitrogen ratios in southern Chilean forest ecosystems}

Published online: 19 August 2004

(C) Springer-Verlag 2004

\section{Oecologia (2004) 140:617-625}

In the column head of Table 1 , the unit for $\mathrm{N}_{\mathrm{a}}$ should be $\mathrm{mg} / \mathrm{kg}$ and not $\mathrm{kg} / \mathrm{ha}$.

Table 1 Structural characteristics and soil variables of lowland successional stands in Chiloé Island. $B A$ Basal area of living trees, $C W D$ coarse woody debris, $N_{a}$ soil content of available N, $N_{\min }$ field

rates of net $\mathrm{N}$ mineralization, $A R A$ potential acetylene reduction activity, $\% W$ soil moisture content

\begin{tabular}{|c|c|c|c|c|c|c|c|c|c|c|}
\hline $\begin{array}{l}\text { Successional } \\
\text { group }\end{array}$ & $\begin{array}{l}\text { Main type of } \\
\text { disturbance }\end{array}$ & $\begin{array}{l}\text { Minimum } \\
\text { stand age }\end{array}$ & $\begin{array}{l}\text { BA } \\
\left(\mathrm{m}^{2} / \mathrm{ha}\right)^{\mathrm{a}}\end{array}$ & $\begin{array}{l}\text { CWD } \\
\text { (ton/ha) }^{\mathrm{a}}\end{array}$ & $\begin{array}{l}\mathrm{N}_{\mathrm{a}} \\
(\mathrm{mg} / \mathrm{kg})^{\mathrm{b}}\end{array}$ & $\begin{array}{l}\mathrm{N}_{\min } \\
(\mathrm{kg} / \text { ha/year })^{\mathrm{c}}\end{array}$ & $\begin{array}{l}\text { ARA } \\
\left(\text { nmol C }_{2} \mathrm{H}_{4} / \mathrm{g}\right. \\
\text { DW/day) }\end{array}$ & $\begin{array}{l}\mathrm{N}_{\text {fix }} \\
(\mathrm{kg} \mathrm{N} / \text { ha/year })^{\mathrm{b}}\end{array}$ & $\begin{array}{l}\text { Soil } \\
\mathrm{C} / \mathrm{N}\end{array}$ & $\% \mathrm{~W}^{\mathrm{c}}$ \\
\hline Early (ES-1) & Fire-logging & $2 \pm 0$ & 1.9 & 62 & 82.9 & 31.4 & 0.68 & 0.22 & 27.0 & 78.0 \\
\hline Early (ES-2) & Fire-logging & $11 \pm 2$ & 3.9 & 42 & 58.6 & 22.0 & 2.65 & 0.11 & 32.6 & 74.1 \\
\hline Mid (MS-1) & Fire-logging & $34 \pm 6$ & 16.6 & 9 & 76.7 & 15.4 & 32.98 & 0.64 & 30.3 & 81.7 \\
\hline Mid (MS-2) & Fire-logging & $51 \pm 58$ & 46.0 & 34 & 57.6 & 62.0 & 0.56 & 0.61 & 21.7 & 69.6 \\
\hline Mid (MS-3) & Fire-logging & $62 \pm 44$ & 49.7 & 36 & 57.7 & 38.0 & 1.28 & 1.66 & 28.2 & 72.4 \\
\hline Late (LS-1) & Selective logging only & $133 \pm 46$ & 65.5 & 47 & 86.6 & 52.4 & 0.87 & 1.41 & 21.8 & 76.9 \\
\hline Late (LS-2) & Selective logging only & $134 \pm 78$ & 85.7 & 65 & 67.7 & 33.2 & 1.3 & 0.23 & 28.3 & 74.7 \\
\hline
\end{tabular}

${ }^{a}$ BA (from Aravena et al. 2002) and biomass of CWD (from Carmona et al. 2002) for each stand

${ }^{\mathrm{b}}$ For the first $10 \mathrm{~cm}$ of soil

${ }^{\mathrm{c}}$ For $0-30 \mathrm{~cm}$ soil depth

${ }^{\mathrm{d}}$ Estimated for surface $(0-10 \mathrm{~cm})$ sieved mineral soil

The online version of the original article can be found at http://dx. doi.org/10.1007/s00442-004-1627-y

C. A. Pérez $(\bowtie) \cdot M$. R. Carmona · J. J. Armesto

Centre for Advanced Studies in Ecology and Biodiversity,

Pontificia Universidad Católica de Chile,

Casilla 114-D

Santiago, CP6513677, Chile

e-mail: cperez@bio.puc.cl

Fax: +56-2-6862621

M. R. Carmona · J. C. Aravena · J. J. Armesto Laboratorio de Sistemática y Ecología Vegetal, CMEB,

Facultad de Ciencias, Universidad de Chile,

Casilla 653,

Santiago, Chile 